INPLASY

PROTOCOL

To cite: Hernández-López et al. Humor and social competence in High School and University education: a systematic review. Inplasy protocol 2021110033. doi:

10.37766/inplasy2021.11.0033

Received: 10 November 2021

Published: 10 November 2021

Corresponding author:

Luis Pablo Hernández-López

luisphernandez@correo.ugr.es

Author Affiliation:

University of Granada.

Support: None.

Review Stage at time of this submission: Risk of bias assessment - Completed but not published.

Conflicts of interest:

None declared.

\section{Humor and social competence in High School and University education: a systematic review}

Hernández-López, LP1; Romero-López, M²; García-Quirante, G³.

Review question / Objective: Research question: What type of relationship exists between the use of humor and social competence, or any of their respective components, in postcompulsory education students? The aim of this paper was to conduct a systematic theoretical review of the relationship between humor and social competence in post-compulsory education students.

Information sources: Electronic bibliographic databases of Psychology, Biomedical and Multidisciplinary Sciences, as well as the ProQuest search platform and the SCOPUS and Web of Science meta-search engines. The electronic databases used were MEDLINE, ProQuest Dissertations \& Theses Global, Psychology Database, APA PsycArticles, APA Psyclnfo, SCOPUS and Web of Science Core Collection. Platforms: ProQuest and the meta-search engines SCOPUS and Web of Science.

INPLASY registration number: This protocol was registered with the International Platform of Registered Systematic Review and Meta-Analysis Protocols (INPLASY) on 10 November 2021 and was last updated on 10 November 2021 (registration number INPLASY2021110033).

\section{INTRODUCTION}

Review question / Objective: Research question: What type of relationship exists between the use of humor and social competence, or any of their respective components, in post-compulsory education students? The aim of this paper was to conduct a systematic theoretical review of the relationship between humor and social competence in post-compulsory education students.

Rationale: Despite all the research that relates humor and social competence, it cannot yet be said that we can establish conclusive and substantiated explanations on the topic, since we do not yet have solid 
and well-founded evidence. For this reason, it has been considered necessary to review the scientific literature, systematize and organize the findings found in the most current studies in order to be able to propose clear explanations and definitive conclusions.

Condition being studied: The aim of this systematic review was to analyze the relationship between humor and social competence in in high school and college students.

\section{METHODS}

Search strategy: The inclusion criteria selected were: (i) population of postcompulsory education students; (ii) studies with results of relationship between humor and social competence; (iii) experimental and observational empirical studies; (iv) original full-text scientific journal articles written in English, French, German, Italian or Spanish. Exclusion criteria referred to studies that could not be included in the inclusion criteria. The search platforms were ProQuest and the meta-search engines SCOPUS and Web of Science.

Participant or population: High school and college students.

Intervention: None.

Comparator: None.

Study designs to be included: Descriptive observational studies.

Eligibility criteria: Empirical experimental and observational studies (descriptive or analytical).

Information sources: Electronic bibliographic databases of Psychology, Biomedical and Multidisciplinary Sciences, as well as the ProQuest search platform and the SCOPUS and Web of Science meta-search engines. The electronic databases used were MEDLINE, ProQuest Dissertations \& Theses Global, Psychology Database, APA PsycArticles, APA Psyclnfo, SCOPUS and Web of Science Core
Collection.Platforms: ProQuest and the meta-search engines SCOPUS and Web of Science.

Main outcome(s): An initial search revealed 445 titles. These titles were organized in reference management software (Mendeley Desktop, PDFNet SDK copyright ( PDFTron Systems Inc., New York, NY, USA). Duplicates (82 references) were removed automatically and records marked as illegible (2 references) manually. The records were then screened by evaluating titles and abstracts. The final result of this process was 14 studies. A Microsoft Excel table (Microsoft Corporation, Readmon, WA, USA) was then designed to re-evaluate the extracted full-text results. The exclusion criteria for each discarded article were specified in the table. Figure 1 shows the flow diagram of articles at each stage of the selection process for the 4 included studies.

Quality assessment / Risk of bias analysis: The four included studies scored $\mathbf{7 2 . 2 2} \%$ on the Q-SSP (Quality Assessment Checklist for Survey Studies in Psychology) (Protogerou \& Hagger, 2020), which is considered acceptable quality. The study assessment process was conducted independently by two authors (L.P.H. and M.R.). In the final process, a meeting was organized in which comparisons were made and disagreements were resolved with the third author (G.G.).

Strategy of data synthesis: The characteristics of the four studies included in the results are shown in Table 2. The four articles finally selected in the review were observational and descriptive. In addition, all of them presented a positive relationship between humor, or one of its styles, and social competence. In one of them, social competence functions as a moderator between humor and social support.

Subgroup analysis: Study Methodology and sample Measuring instruments Summary of results Statistical analysis Statistical parameters.

Language: English. 
Country(ies) involved: Spain.

Keywords: humor; humour; sense of humor; social competence; socialemotional competence; interpersonal competence.humor; sentido del humor; competencia social; competencia socioemocional; competencia interpersonal.

Contributions of each author:

Author 1 - Luis Pablo Hernández-López Directed the project, drafted and revised the original manuscript, and performed the methodological assessment.

Email: luisphernandez@correo.ugr.es

Author 2 - Miriam Romero-López - Directed the data search, drafted and revised the original manuscript, and performed the methodological assessment.

Email: miriam@ugr.es

Author 3 - Guillermo García-Quirante Directed the data search, drafted and revised the original manuscript, and performed the methodological assessment. Email: guille93g@correo.ugr.es 